\title{
INOVAÇÕES SOCIAIS E SEUS NÍVEIS DE ANÁLISE: O CASO PATAC - PB
}

SOCIAL INNOVATIONS AND THEIR ANALYSIS LEVELS:

THE PATAC - PB CASE

Suzanne Érica Nóbrega Correia

Doutora em Administração pela Universidade de Pernambuco (Recife/Brasil) Professora na Universidade Federal de Campina Grande (Campina Grande/Brasil) E-mail:suzanne.enc@gmail.com

Carla Regina Pasa Goméz Doutora em Administração pela Universidade do Rio Grande do Sul (Porto Alegre/Brasil) Professora na Universidade de Pernambuco (Recife/Brasil) E-mail: carlapasagomez@gmail.com
Verônica Macário de Oliveira Doutora em Administração pela Universidade de Pernambuco (Recife/Brasil) Professora na Universidade Federal de Campina Grande (Campina Grande/Brasil) E-mail:veronicamacario@gmail.com 


\section{RESUMO}

Este artigo tem como objetivo analisar as iniciativas de inovação social desenvolvidas pelo Programa de Articulação de Tecnologias Apropriadas às Comunidades (PATAC), a partir do modelo proposto por Lévesque (2002). Nesse sentido, foram observados os três níveis de análise apresentados no modelo: atores, organizacional e institucional. Realizou-se um estudo de caso descritivo e exploratório, a partir da realização de entrevistas semiestruturadas junto a gestores e técnicos do programa supracitado. Na análise dos atores, percebe-se envolvimento da comunidade pela valorização da cultura e da capacidade de aprendizado. No nível organizacional, observou-se que, nos projetos geridos, tem um caráter compartilhado, na busca do fortalecimento sociopolítico. Os resultados demonstram que, no nível institucional, o processo de inovação social tem como função coordenar os diferentes setores da sociedade, para manter redes de relacionamentos e cooperação para funcionamento dos projetos e programas sociais envolvidos. Assim, os níveis de análise da inovação social não devem ser entendidos apenas como mecanismo isolado de satisfação das necessidades sociais, mas também como um mecanismo de transformação social que busca mudanças na configuração da sociedade como meio de promover a sustentabilidade e a qualidade de vida.

Palavras-chave: Inovação social. Níveis de análise da inovação social. Desenvolvimento local.

\section{ABSTRACT}

This paper aims to analyze the social innovation initiatives developed by the Appropriate Technology Joint Program for Communities (PATAC) from the model proposed by Lévesque (2002). In this sense, the three levels of analysis presented in the model were observed: actors, organizational and institutional. We conducted a study of descriptive and exploratory case from conducting semi-structured interviews with managers and technicians of the aforementioned program. In the analysis of the actors, the community's involvement is appreciated by the valorization of the culture and the learning capacity. At the organizational level, it was observed that in managed projects, it has a shared character, in the search for socio-political strengthening. The results demonstrate that at the institutional level the social innovation process has the function of coordinating the different sectors of society to maintain networks of relationships and cooperation for the operation of the social projects and programs involved. Thus, analysis of levels of social innovation should not be understood only as a single mechanism of satisfaction of social needs, but also as a social transformation engine that seeks changes in society setting as a means of promoting sustainability and quality of life.

Keywords: Social innovation. Analysis of levels of social innovation. Local development. 


\section{INTRODUÇÃO}

Os problemas decorrentes do modelo de desenvolvimento focado no crescimento econômico têm sido enfrentados e discutidos em diferentes perspectivas, contemplando, total ou parcialmente, os aspectos econômicos, sociais e ambientais relacionados à sobrevivência das sociedades. Isto fez emergir o interesse de pesquisadores em buscarem compreender as questões que envolvem a configuração de tal modelo e a proporem novas formas de organização de empresas, comunidades e sociedades que contemplem as mudanças impostas pelas dinâmicas ambientais, sociais e de mercado.

Essas novasformas de organização são caracterizadas como uma construção social do desenvolvimento, que incita a sociedade civil a trocar experiências, mudar comportamentos e gerar soluções na busca por atender as suas necessidades e a promover uma sociedade mais justa e equitativa. O entendimento de que é necessário buscar investimentos para uma mudança social é o início do reconhecimento de que os desafios futuros podem ser identificados e de que existem possíveis soluções que podem ser implementadas. Assim, estratégias de mobilização e de envolvimento das comunidades no processo de mudança são centrais na proposta de um desenvolvimento mais sustentável, pressupondo ampla participação social desde a elaboração de um diagnóstico local até a formulação, implementação e monitoramento das ações (BODSTEIN et al., 2004; MOULAERT, 2009). Para tanto, faz-se necessário conceber estratégias inovadoras que contemplem diferentes vertentes da estrutura da sociedade e que possam contribuir com o processo de criação, aprendizagem e intervenção da realidade. A inovação social (IS) surge, nesse contexto, como uma possibilidade para auxiliar as comunidades a implantarem programas de melhorias no seu entorno, a desenvolverem produtos de impacto social significativo e, ainda, a criar e difundir novas tecnologias sociais adaptadas às especificidades de cada comunidade.

Para um melhor entendimento de como este processo ocorre, é necessário compreender a definição que está atrelada ao termo "inovação social" que a classifica como uma abordagem que busca novas respostas para os problemas sociais, no que tange à implementação de novos processos de integração no mercado de trabalho, novas competências e novas formas de participação, bem como ao estabelecimento de uma ligação explícita com o desenvolvimento local e com a melhoria da qualidade de vida (OECD, 2010).

Para atingiros seus propósitos, as inovações sociais precisam sefortalecer. Isto requero desenvolvimento de metodologias de pesquisa e desenvolvimento no setor público, com foco no fomento às iniciativas e à implementação de ferramentas e do empoderamento dos atores sociais para atuarem como representantes e gestores das inovações (MOULAERT et al., 2005), a exemplo das organizações sociais que são consideradas um campo fértil de possibilidades para a implementação de inovações sociais (MULGAN et al., 2007). Estas organizações buscam responder, de forma inovadora, aos desafios econômicos e sociais atuais, criando postos de trabalho, aprofundando a integração social e a melhoria dos serviços sociais locais (COMISSÃO 
EUROPÉIA, 2011), atuando como agentes das transformações sociais, políticas e econômicas dentro de suas localidades de modo que possam auxiliar a promover o desenvolvimento sustentável.

Neste contexto, faz-se mister dispor de mecanismos de investigação que possibilitem entender como as inovações sociais ocorrem no contexto das organizações sociais, para capturar as suas características essenciais, diante das seguintes necessidades: 1) de se ter uma resposta concreta, que sirva como referência confiável para a formação de argumentos, para apoiar os atores envolvidos no processo de tomada de decisões em programas de IS; 2) de operacionalização do termo inovação social que consiga ligar pressupostos teóricos à aspectos empíricos; 3) e de oferecer um suporte às organizações específicas envolvidas nessas iniciativas (EVA et al., 2013). Uma dessas ferramentas é o modelo desenvolvido por Lévesque (2002) que compreende as inovações sociais como uma nova configuração do capitalismo, segundo três níveis de análise: atores, organizacional e institucional.

Assim, este artigo tem como objetivo analisar as iniciativas de inovação social desenvolvidas pelo Programa de Articulação de Tecnologias Apropriadas às Comunidades (PATAC), a partir do modelo proposto por Lévesque (2002). O PATAC é baseado no desenvolvimento das potencialidades e interesses das populações locais, especialmente os agricultores familiares, em questões relacionadas à conservação, uso sustentável e recomposição ambiental de seus recursos naturais. Busca contribuir para a implementação de ações integradas para o semiárido, fortalecer as inserções políticas, técnicas e organizacionais demandadas pelas instituições atuantes em nível local e apoiar a difusão de métodos, técnicas e procedimentos que contribuam para o processo de aprendizagem social construir um território inteligente.

Para tanto, em termos metodológicos, realizou-se um estudo de caso de natureza descritiva e exploratória, a partir da realização de entrevistas semiestruturadas junto a gestores e técnicos do programa supracitado.

Este artigo está organizado em cinco seções. Além desta introdução, é apresentado na seção dois a perspectiva teórica da inovação social e suas formas de abordagem, bem como o modelo de avaliação da Inovação Social proposto por Lévesque (2002). Na terceira seção, descreve-se o percurso metodológico adotado para a realização deste estudo. Na sequência, os resultados são apresentados com base na aplicação do modelo proposto e, ao final, são discutidas as considerações finais dos autores.

\section{INOVAÇÃO SOCIAL}

Na década de 70, o CRISES (Centre de Recherche sur les innovations sociales), como centro de estudos interuniversitário que reúne pesquisadores de oito instituições de ensino superior do Québec, passou a questionar a relação exagerada entre as inovações tecnológicas e as inovações sociais (LÉVESQUE, 2002). Então, o CRISES passou a focar seus estudos na busca de pontes entre o desenvolvimento econômico e o 
desenvolvimento social, estabelecendo uma ligação entre as inovações sociais (consideradas como nível micro) e transformações sociais (consideradas como nível macro), usando como ambiente de estudo o Modelo do Quebec. A partir desses estudos, os conceitos gerados de inovação social tenderam a se afastar da literatura especializada em inovação, incluindo as contribuições neo-schumpterianas e evolucionistas (LÉVESQUE, 2002).

Os estudos sobre inovação social discutem alternativas de crescimento e desenvolvimento das comunidades e dos indivíduos. A questão central foca-se na busca da realização e das potencialidades dos indivíduos, de um melhor nível de qualidade de vida e bem-estar e na busca pela felicidade e realização desses indivíduos como pessoas humanas (CARON, 2007).

Neste contexto de mudança, as inovações sociais estão interessadas no surgimento de uma nova configuração do capitalismo cujos contornos permanecem mudando com base nos seguintes aspectos: 1) no nível do desenvolvimento social, encontrando novos atores, incluindo a sociedade civil, organizações não governamentais, entre outros; 2) no sistema político, através de uma reconfiguração de poder entre os Estados-nações, organismos mundiais, autoridades locais e regionais; e 3) em formas organizacionais, nas novas formas de coordenação e governança, não apenas redefinindo a relação entre o desenvolvimento social e econômico, mas também entendendo melhor o que é a economia social (LÉVESQUE, 2002).

Observa-se assim, que o conceito de inovação social se torna amplo e extenso, com foco em uma atitude crítica e um desejo de mudança (seja através de ação deliberada, intencional ou voluntária), que culmina em um processo de emancipação e produção de conhecimento pelos próprios atores envolvidos.

Pol e Ville (2009) acreditam que é necessário estabelecer a inovação social como um campo de pesquisa respeitável, amplo e útil que acomoda significativos casos empíricos. Assim, os autores propõem uma definição de inovação social, sem declarar o que "é", embasada na criação de novas ideias exibindo o impacto positivo na qualidade e quantidade de vida dos indivíduos.

Para a geração da inovação social três requisitos devem ser atendidos, a saber: 1) satisfação das necessidades humanas, não atendidas pelas vias convencionais, ou seja, o mercado; 2) como resultado, a promoção da inclusão social; e 3) a capacitação de atores sociais sujeitos, potencial ou efetivamente, a risco de exclusão/marginalização social, proporcionando uma mudança mais ou menos intensa das relações de poder (ANDRÉ; ABREU, 2006).

Assim, a inovação social está profundamente preocupada com a transformação da sociedade e para a melhoria da vida humana, portanto, a teoria envolvida não deve ser usada apenas para interpretar a inovação social como um conjunto de fenômenos, mas também para mobilizar e orientar a prática de transformação e entender as condições sociais e institucionais em que estão envolvidas (MOULAERT et al., 2010; BOCK, 2016).

Percebe-se que a inovação social se volta para a criação de valor, para os interesses dos grupos sociais e da comunidade. Apresenta-se como uma nova resposta a uma situação não satisfatória, e visa o bem-estar 
dos indivíduos e das coletividades através do atendimento a necessidades como saúde, educação, trabalho, lazer, transporte e turismo (CLOUTIER, 2003; BITTENCOURT; RONCONI, 2016).

A inovação social é entendida como um processo de transformação nos padrões de resposta a necessidades sociais profundas, através da ruptura com as normas vigentes, com os valores instituídos e com a estrutura da distribuição de poder e recursos. Isto é, a inovação surge como uma inciativa que escapa à ordem estabelecida, implicando em uma mudança clara, criativa e efetiva, na forma como uma sociedade lida com um determinado problema social complexo, anteriormente sem respostas contundentes, como a pobreza, a violência ou a deterioração ambiental (NILSSON, 2003; HOWALDT; DOMANSKI; KALETKA, 2016).

O enfoque está, sobretudo, no âmbito dos processos - inclusão, capacitação e cooperação; uma vez que, a própria proposta de mudança social, objetivo último desta articulação de estratégias, se situa a nível processual (ANDRÉ; ABREU, 2006).

Dentro do processo de inovações sociais, são enfatizadas as estratégias de vinculação permanente e de cooperação intensa entre os atores envolvidos no sentindo de se obterem transformações sociais duradouras e de impacto, que possam representar mudanças nas relações e nas condições sociais (BIGNETTI, 2011). É um processo de construção social onde os grupos sociais se envolvem no desenvolvimento de uma solução dentro de uma realidade de contexto e que se chega a uma situação de "estabilização" e "fechamento" (DAGNINO; BRANDÃO; NOVAES, 2009).

A inovação social se volta para as ações comunitárias e geralmente abrange pequenos esforços e localidades devido às dificuldades de se alavancar recursos (BIGNETTI, 2011). Promove novas ações que valorizam o progresso de uma sociedade local, oferecem respostas a um conjunto de questões relacionadas à saúde, educação, habitação, segurança, e às necessidades de melhorar a partir de ideias às ações inovadoras. No contexto local, as iniciativas de inovação social propiciam novas práticas a favor do desenvolvimento econômico, do aproveitamento de recursos e estímulo à produtividade, à formação do capital social e do redesenho da arquitetura institucional, em sentidos favoráveis à ampliação dos espaços da governabilidade democrática e a participação dos cidadãos (RUIZ, 2012).

A caracterização da inovação social pode ser associada a dois processos, o primeiro voltado para a transformação das estruturas de gestão pública como forma de inclusão dos interesses dominados na esfera pública, para além da forma tradicional de representação; e o segundo focado na geração de redes associativas, capaz de gerar processos sinérgicos entre as instituições estatais democratizadas e as organizações da sociedade (GENRO, 1997).

Lassance Jr e Pedreira (2004) corroboram, afirmando que a inovação social parte do pressuposto da possível articulação de uma ampla rede de atores; que precisam ser estruturadas em modelos flexíveis quando se pensa em adaptação dessas tecnologias; e que cumprem quatro fases essenciais: a de criação, 
de viabilidade técnica, de viabilidade política, de viabilidade social; e que para se chegar à situação de estabilização e fechamento, é necessária a articulação entre governo, administração, especialistas e organizações sociais.

Com efeito, uma organização social é uma empresa cujo objetivo principal é ter incidência social, fornecendo bens e prestação de serviços de caráter social, gerida de forma responsável e transparente, de interesse comum, e com alto nível de inovação social; ser essencialmente sem fins lucrativos e com excedentes reinvestidos nos projetos sociais; e seu modo de organização baseia-se em princípios democráticos ou participativos, visando à justiça social (COMISSÃO EUROPEIA, 2011; HOWALDT; KOPP; SCHWARZ, 2015).

A inovação social é estritamente ligada ao processo das organizações sociais no que se refere à conscientização dos seus espaços e influências junto aos agentes na elaboração, implementação e prestação do serviço e bem-estar público. Neste contexto, é possível afirmar que organizações sociais têm contribuído para o fortalecimento de iniciativas de inovação social. Relacionadas a esse contexto, as inovações sociais emergem como fator de renovação dos serviços de bem-estar e na contribuição para a mudança social, estando ligadas às estruturas democráticas e participativas de governo (HULGARDI; FERRARINI, 2010).

Assim, organizações sociais desempenham uma força motriz para a inovação social, na introdução de novos métodos para a execução de serviços, na criação e implementação de tecnologias sociais, e na intervenção para melhoria da qualidade de vida dos indivíduos. Existem também dimensões de análise utilizadas para caracterizar o modelo de desenvolvimento baseado na relação entre os atores sociais, as instituições e os sistemas organizacionais, apresentado de forma mais detalhada na próxima subseção.

\subsection{NÍVEIS DE ANÁLISE DA INOVAÇÃO SOCIAL: MODELO DE LÉVESQUE (2002)}

Uma inovação pode ser considerada social por, no mínimo, duas razões: 1) através da satisfação das necessidades humanas não atendidas e; 2) nas relações sociais entre indivíduos e grupos de determinada localidade (MOULAERT et al., 2005; SHARRA; NYSSENS, 2010; CAJAIBA-SANTANA, 2014; BITTENCOURT; RONCONI, 2016). Assim, os conceitos de inovação social integram duas perspectivas, uma com foco no resultado e outra com foco no processo (SHARRA; NYSSENS, 2010; HULGARD; FERRARINI, 2010; CORREIA; OLIVEIRA; GOMÉZ, 2016).

Na perspectiva da inovação social como resultado, os conceitos adotados enfatizam a necessidade de solucionar problemas para situações não atendidas, como também na criação de valor que é gerado para a sociedade como um todo (MULGAN et al., 2007; PHILLS JR.; DEIGMEIER; MILLER, 2008).

A inovação social dentro da perspectiva do processo, não visualiza apenas a necessidade social que não está sendo atingida, mas as alternativas de inovação social que surgem a partir das condições específicas 
no contexto socioeconômico de uma determinada localidade (SHARRA; NYSSENS, 2010). A inovação social surge como uma reação às pressões resultantes de necessidades não atendidas, seja por movimentos sociais, representantes da sociedade civil ou organizações não governamentais, e respondem a uma condição de necessidade específica.

Outra caraterística da inovação social como processo é a formação de arenas colaborativas caraterizadas por uma complexa rede de parcerias formais e informais entre vários atores envolvidos (SHARRA; NYSSENS, 2010; HULGARD; FERRARINI, 2010).

Assim, a inovação social como um processo pode ser classificada em quatro etapas (MULGAN et al., 2006), a saber: 1) a descoberta de uma necessidade não atendida, juntamente com a ideia de como poderia ser cumprida, na qual as novas possibilidades podem ter caráter tecnológico, organizacional ou ser resultado de novos conhecimentos; 2) testa-se a viabilidade da inovação social na prática, gerando um encontro com a realidade, para minimizar as incertezas e realizar ajustes para melhorar seus produtos ou serviços inovadores; 3) o protótipo passa a ser cultivado e replicado em larga escala, captando-se recursos e parcerias institucionais para facilitar a expansão da atividade; e 4) por fim, enfatiza-se a aprendizagem e adaptação contínua no contexto adotado. Essas quatro etapas apresentadas facilitam o entendimento de como funciona o processo da inovação social.

Lévesque (2002) apresenta as inovações sociais como uma nova configuração do capitalismo, segundo três níveis de análise: 1) inovações sociais centradas nos atores; 2) inovações sociais em nível organizacional; 3) inovações sociais em nível institucional. Estes três níveis são considerados interdependentes pelo grau de relação que possuem entre si e que não podem ser entendidas como um fator limitador, mas como uma orientação primeira do impacto da inovação social (RODRIGUES, 2012). A Figura 1 apresenta os aspectos inseridos em cada um desses níveis. 
Figura 1 - Níveis de análise da inovação social

\begin{tabular}{|c|c|c|}
\hline \multicolumn{3}{|c|}{ NÍVEIS DE ANÁLISE DA INOVAÇÃO SOCIAL } \\
\hline ATORES & ORGANIZACIONAL & INSTITUCIONAL \\
\hline $\begin{array}{l}\text { Formas de cooperação e } \\
\text { geração de aprendizagem } \\
\text { Novos atores sociais } \\
\text { Novos papéis sociais } \\
\text { Grau de inclusão de } \\
\text { usuários beneficiados }\end{array}$ & $\begin{array}{l}\text { Novas formas de divisão } \\
\text { e coordenação do trabalho } \\
\text {. Novas configurações } \\
\text { organizacionais } \\
\text {. Formas de utilização dos } \\
\text { recursos } \\
\text { Grau de participação dos } \\
\text { atores }\end{array}$ & $\begin{array}{l}\text { Relação intersetorial } \\
\text { Grau de valor social } \\
\text {. Universalização dos } \\
\text { direitos }\end{array}$ \\
\hline
\end{tabular}

Fonte: Levésque (2002) e Rodrigues (2012)

A inovação social com foco no indivíduo refere-se à cooperação entre os atores sociais envolvidos para a criação, produção e difusão da inovação, sendo assim, o surgimento de inovações sociais é resultante da criação de equipes multidisciplinares (TAYLOR, 1970; CLOUTIER, 2003). A criação dessas novas relações sociais dá suporte na mediação individual e coletivizada, concebida não só para resolver problemas sociais, mas também para responder a um ideal social.

Na medida em que acontece a reestruturação da rede de atores sociais, é provocada uma redefinição de orientações culturais que formaliza a adoção da nova gestão das relações sociais e redireciona o estabelecimento de novas formas de fazer as coisas, seja através do desenvolvimento de novos serviços, processos, produtos ou novas formas de organização das relações sociais (FONTAN, 1998; TARDIF; HARRISSON, 2005; KLEIN; HARRISSON, 2007; ASSOGBA, 2010; HOWALDT; KOPP; SCHWARZ, 2015; BOCK, 2016).

As inovações sociais envolvem as pessoas no centro do processo para enriquecer as políticas sociais, os projetos de desenvolvimento nacionais e locais e desenvolver parcerias (LALLEMAND, 2001). Portanto, a inovação social se distingue principalmente pelo processo ancorado na participação dos atores envolvidose partes interessas da sociedade civil e na qualidade dessas parcerias realizadas.

A importância gerada na participação dos indivíduos para a inovação social está centrada, principalmente, no processo de aprendizagem para aquisição de conhecimentos, mudança de representações, de novos aprendizados e cooperação (LEVÉSQUE, 2002; CLOUTIER, 2003). A participação ativa dos atores é que define todo o escopo do projeto de inovação social através de um processo de aprendizagem e que finaliza com a resolução dos problemas. 
Vantagens do crescimento e desenvolvimento dos atores são apresentadas a partir de uma visão integrada, considerando diferentes níveis dos fatores que contribuem para a existência da inovação social, bem como a dinâmica de inter-relação entre esses atores. Outro fator relevante é a conscientização e a capacitação dos atores no processo de resolução de problemas, uma vez que são considerados como agentes de mudança autônomos e os principais responsáveis por diagnosticar as necessidades sociais e pela resolução de problemas (LEVÉSQUE, 2002; CLOUTIER, 2003).

Assim, o processo de criação e de implementação é desenvolvido a partir da consciência e participação dos atores envolvidos, que são considerados participantes efetivos ao longo do processo. A condição essencial para a existência da inovação social é a participação, autonomia e desejo de mudança. Os atores são os protagonistas da inovação social, e ela envolve uma diversidade de atores, entre eles representantes da sociedade civil, do mercado e do governo. Mesmo que existam interesses diferentes entre os atores, deve sempre existir um canal de conciliação e ajustamento, cada um assumindo seu papel (CHAMBON; DAVID; DEVEVEY, 1982; DAVIES et al., 2012; CORREIA; OLIVEIRA; GOMÉZ, 2016).

A inovação exige um bom conhecimento de todos os atores envolvidos no processo. Os fatores de sucesso na implementação de uma inovação estão na sinergia dos atores e sua motivação para inovar, na força para lidar com os obstáculos ena disponibilidade de gerenciar os recursos para sanar os obstáculos (TARDIFF; HARRISSON, 2005). A inovação social assume, então, uma participação renovada dos atores que ocupam papéis diferenciados distribuídos em várias esferas, com diferentes culturas e identidades em busca de um interesse coletivo e público.

As inovações sociais no nível organizacional fazem parte do desenvolvimento das estruturas de produção e referem-se a novas formas de organização do trabalho, podendo ser dividida em duas perspectivas analíticas: a primeira, instrumental, onde gera a reorganização do trabalho como determinante na capacidade de inovar, e discute a questão do bem-estar dos colaboradores; a segunda, a não instrumental, considera novas formas de organização do trabalho como inovação social, já que estas podem melhorar a qualidade de vida no trabalho (CLOUTIER, 2003).

A inovação social, dentro desta perspectiva, tem como objetivo uma (re)organização do trabalho, que envolve uma nova divisão de estrutura de poder, a reorganização do tempo do trabalho, aumentar a autonomia dos funcionários, favorecer o empoderamento e valorizar a pessoa humana (CLOUTIER, 2003; BIGNETTI, 2011).

A inovação social é entendida como a produção de novas ideias, de novas estruturas e de um processo de re-contextualização onde são socialmente (re)construídas as normas do bem público, da justiça e da equidade (CAULIER-GRICE et al., 2012). Demonstra uma construção de mudança social que enfatiza o conhecimento e bens culturais das comunidades e coloca em primeiro plano a reconfiguração coletiva das relações sociais (CAULIER-GRICE et al., 2012). 
A criação e implementação de inovações sociais resulta em mudanças significativas nos atores envolvidos, portanto, esta abordagem integrada exige uma reorganização no trabalho através de uma equipe interdisciplinar, introdução de novas tarefas, nova divisão do trabalho com base nas aptidões e interesses das partes, novos modos de coordenação e um novo aprendizado para melhorar a prática (CLOUTIER, 2003).

Desta forma, envolve mudança de atitudes, comportamentos ou percepções de um grupo de pessoas que, através de uma rede de interesses alinhados, levam a novas e melhores maneiras de ação colaborativa no seio do grupo (CAULIER-GRICE et al., 2012). Assim, uma inovação social acontece quando um grupo de atores muda sua maneira de fazer as coisas e isso leva a uma melhoria significativa para esse grupo.

Logo, o modo como os atores interagem e coordenam o desenvolvimento de uma inovação social gera um potencial nos indivíduos, através de um processo de aprendizagem para aquisição dos conhecimentos, mudança de representações, novos aprendizados e um sistema de cooperação.

A inovação social é uma questão de inovação de processo, de mudanças na dinâmica das relações sociais e suas relações de poder que levam a uma maior inclusão dos indivíduos às várias esferas da sociedade, refere-se a uma posição de justiça social como resultado de uma construção social.

As ações de inovação social estão orientadas para mudarem uma situação social insatisfatória, reunindo vários atores sociais, tais como indivíduos, governos, empresas, comunidade, organizações da sociedade civil que vinculam com o objetivo de reformular os papéis sociais (RODRIGUES, 2004).

No nível de análise orientada para o sistema institucional, estão incluídos os sistemas de regras, a partilha de direitos e responsabilidades, sistemas de gestão de conflitos e o sistema político. Considera-se que, a partir da mobilização dos atores sociais, o ambiente institucional pode se transformar. Ou seja, as instituições são transformadas pelas demandas de participação ou de inclusão de atores antes não atendidos pelas ações governamentais ou por políticas públicas (LÉVESQUE, 2002).

Neste contexto, o conceito de inovação social adotado está associado à transformação social, ou seja, criação de novos objetivos econômicos e sua regulamentação, proteção do meio ambiente e garantia da sustentabilidade dos recursos naturais, um novo papel na espera política, a exemplo da descentralização e a cooperação entre diferentes grupos, e das possibilidades de cooperação a nível internacional (CLOUTIER, 2003).

Em suma, é um conceito que abrange tanto as soluções inovadoras geradas a partir dos problemas econômicos e sociais identificados, quanto à cooperação entre os diversos atores envolvidos durante a fase de criação e implementação da inovação social.

Portanto, as ações de inovação social que contemplam essas categorias de análise geram melhores índices de sustentabilidade, contemplando um modelo de desenvolvimento com integração econômica, social e ambiental, visando promover a justiça social e preservação ambiental (OLIVEIRA; SILVA, 2012). 
Constitui-se, assim, a inovação social como um poderoso instrumento de integração social, com o objetivo de fortalecer a sociedade civil para enfrentar problemas de natureza socioambiental e econômica, viabilizando atividades de produção, prestação de serviços, comercialização e consumo mais sustentável.

\section{PROCEDIMENTOS METOdOLÓGICOS}

Esta pesquisa foi concebida com o objetivo de analisar as iniciativas de inovação social desenvolvidas pelo Programa de Articulação de Tecnologias Apropriadas às Comunidades (PATAC), a partir do modelo proposto por Lévesque (2002). Desta forma, definiu-se que o método mais adequado para a condução desta pesquisa é o Estudo de Caso, considerando que uma investigação deste tipo deve se apresentar em diversas fontes de evidências, baseando-se em desenvolvimento prévio de proposições teóricas que conduzam a uma adequada coleta e análise dos dados (YIN, 2005).

Em termos de métodos de procedimentos, este estudo caracteriza-se como exploratório e descritivo. Exploratório por ter como finalidade proporcionar maiores informações sobre como o processo de inovação social acontece no âmbito das organizações sociais a partir dos níveis de análise propostos por Lévesque (2002), facilitando a delimitação temática do estudo, uma vez que o tema ainda é pouco explorado. Descritivo por exprimir características do programa investigado, delimitando interligações entre os aspectos investigados e a teoria relacionada, definindo suas naturezas e implicações.

A unidade de análise deste estudo foi o Programa de Articulação de Tecnologias Apropriadas às Comunidades (PATAC), que é considerado um exemplo de organização social com foco na inovação social, e tem como objetivo combater a vulnerabilidade da agricultura familiar na região de entorno, através do fortalecimento dos processos de inovação social junto às famílias dos agricultores e suas organizações. Os sujeitos investigados na pesquisa foram os representantes da Coordenação Executiva do PATAC, composta por dois membros, uma coordenadora administrativa e um coordenador técnico, além de três sócios efetivos responsáveis pela implementação e acompanhamento dos projetos.

O PATAC possui um papel de articulador entre os atores envolvidos no processo de inovação social. Está vinculado ao Governo Federal, através da representação das políticas públicas adotadas, assim como os benefícios, mais especificamente, os agricultores familiares, ao contribuir, através da implementação de políticas públicas, para a mudança da realidade na qual esses atores sociais estão inseridos. Vale ressaltar que a unidade de análise investigada é constituída por representações (lideranças locais) da comunidade local, o que torna capaz de avaliar as dimensões da inovação social no contexto no qual está inserida.

A coleta de dados da pesquisa contou com dados primários e secundários. As técnicas utilizadas na coleta de dados foram: entrevistas em profundidade, análise documental e observação não-participante. 
Nas entrevistas realizadas, utilizou-se como instrumento de coleta de dados um roteiro semiestruturado elaborado a partir do modelo de Lévesque (2002) para avaliação de inovações sociais. O conteúdo das entrevistas foi gravado com recursos de áudio, além de anotações em caderneta. A análise documental ocorreu a partir de publicações e relatórios, nos quais constam informações sobre o programa investigado. Por fim, a observação não-participante ocorreu durante as visitas dos pesquisadores em campo para realização das entrevistas.

Os dados foram analisados a partir da utilização da técnica de análise de conteúdo (AC), definida como um conjunto de técnicas de análise que envolve procedimentos sistemáticos e objetivos de descrição de conteúdos das informações coletadas (BARDIN, 2008), pré-análise, exploração de material, tratamento dos dados, inferência e interpretação, a fim de compreender o melhor discurso aplicado entre os entrevistados (RICHARDSON, 1985). Na figura 2, a seguir, apresenta-se a visão geral dos procedimentos metodológicos adotados neste estudo.

Figura 2 - Delineamento metodológico da pesquisa

\begin{tabular}{|c|c|c|}
\hline \multicolumn{3}{|c|}{ Estratégias Metodológicas } \\
\hline $1^{\mathrm{a}}$ Fase & $2^{\mathrm{a}}$ Fase & $3^{\text {a }}$ Fase \\
\hline Pesquisa bibliográfica & Coleta de dados & Análise dos dados \\
\hline $\begin{array}{l}\text { Níveis de análise da } \\
\text { inovação social: } \\
\text {. Atores } \\
\text {. Organizacional } \\
\text {. Institucional }\end{array}$ & $\begin{array}{l}\text { Análise documental } \\
\text {. Entrevista } \\
\text {. Observação não } \\
\text { participante }\end{array}$ & $\begin{array}{l}\text { Pré-análise } \\
\text { Exploração } \\
\text {. Tratamento }\end{array}$ \\
\hline $\begin{array}{c}\text { Análise qualitativa: } \\
\text { categorização dos dados }\end{array}$ & & $\begin{array}{l}\text { Análise qualitativa e } \\
\text { triangulação dos dados }\end{array}$ \\
\hline
\end{tabular}

Fonte: elaborado pelos autores

Esta pesquisa preocupou-se com os critérios de validade e confiabilidade para que o resultado alcançado fosse o mais próximo da realidade do estudo. Os resultados encontrados serão analisados em detalhe na próxima seção. 


\section{ANÁLISE DOS RESULTADOS}

Iniciativas das organizações sociais devem ser estudadas sob a lente da inovação social para que seja possível mapear a extensão, características, impactos e resultados das inovações sociais para o desenvolvimento sustentável. Uma análise em profundidade se faz necessária para compreender as condições para criação e implementação de inovações sociais e as condições para sua difusão, que podem gerar práticas de sustentabilidade significativas para a localidade estudada.

Nesse contexto, este estudo teve como unidade de análise o PATAC (Programa de Articulação de Tecnologias Apropriadas às Comunidades) que é uma organização social que tem como objetivo trabalhar pela viabilidade da agricultura familiar, especialmente no Semiárido Paraibano, buscando fortalecer os processos de experimentação e difusão de inovações técnicas e sócio-organizativas junto aos pequenos agricultores, com foco no desenvolvimento sustentável (PATAC, 2014).

Dos projetos desenvolvidos pelo PATAC, dois são em parceria com a Articulação do Semiárido - ASA: o Programa um Milhão de Cisternas (P1MC), com objetivo de instalar cisternas para retenção de água para o consumo humano; e o Programa Uma Terra e Duas Águas (P1+2), que provê as cisternas de águas para produção de alimentos. Além desses programas, o PATAC desenvolve os fundos rotativos solidários, que demonstram e fortalecem um processo de organização solidária nas comunidades. Tais programas têm bases em tecnologias simples, de baixo custo, fácil difusão e construídas a partir da mobilização das comunidades necessitadas (DAGNINO, 1994).

Para melhor entendimento de como as iniciativas de inovação social ocorrem no PATAC, as subseções seguintes apresentam os níveis de análise propostos no modelo de Lévesque (2002), de acordo com a Figura 3. 
Figura 3 - Níveis de análise da inovação social no caso investigado

\begin{tabular}{|c|c|c|}
\hline \multicolumn{3}{|c|}{$\begin{array}{c}\text { NÍVEIS DE ANÁLISE DA INOVAÇÃO SOCIAL } \\
\text { o caso do PATAC }\end{array}$} \\
\hline ATORES & ORGANIZACIONAL & INSTITUCIONAL \\
\hline $\begin{array}{l}\text { Captação de recursos } \\
\text { através de editais } \\
\text { Constituição de uma } \\
\text { comissão municipal dos } \\
\text { projetos } \\
\text {. Intercâmbio de } \\
\text { experiências bem sucedidas } \\
\text { Emancipação dos atores } \\
\text { locais }\end{array}$ & $\begin{array}{l}\text {. Modelo de gestão integrado } \\
\text { e participativo } \\
\text {. Política democrática junto } \\
\text { a todos os atores envolvidos } \\
\text {. Processo de mobilização, } \\
\text { formação e implementação } \\
\text { das inovações sociais } \\
\text {. Abordagem emancipatória }\end{array}$ & $\begin{array}{l}\text { Processo Bottom-up na } \\
\text { definição de políticas } \\
\text { públicas } \\
\text {. Envolvimento dos atores } \\
\text { sociais, organizacionais e } \\
\text { institucionais } \\
\text {. Legitimidade dos atores } \\
\text { envolvidos }\end{array}$ \\
\hline
\end{tabular}

Fonte: Elaborado pelos autores

\subsection{NÍVEL DOS ATORES}

O conceito de inovação social, já discutido neste artigo, envolve novas soluções que atendam a uma necessidade social, através da articulação de atores que permitam o alcance de um resultado social. Assim, pensar em envolvimento da sociedade civil dentro do conceito de inovação social é compreender as formas como os atores se articulam e como podem se envolver no processo de desenvolvimento de novas soluções para os desafios sociais. A preocupação com o processo de implementação da inovação social volta-se para o envolvimento e até a emancipação dos atores envolvidos, tendo como foco os próprios produtores e usuários dessas inovações (RODRIGUES; BARBIERI, 2008).

Ao buscar o entendimento de como as inovações sociais ocorrem no âmbito das organizações sociais sob a perspectiva do nível de atores, verificou-se que, no PATAC, os recursos para a construção de cisternas surgem através de editais, que já deixam explicitados quais municípios devem ser beneficiados e quantas implementações de cisternas devem ser contempladas, respeitando sempre as especificidades geográficas de cada região. Assim, a definição dos atores que deverão ser inseridos nas iniciativas segue as exigências do Edital que determina que, para execução de cada um dos projetos, o primeiro passo do processo decisório é constituir uma comissão municipal. Esta comissão deve ser composta por membros das organizações sociais, sindicatos, associações de pequenos agricultores, pessoas ligadas a entidades religiosas, representantes 
do conselho municipal e representantes das comunidades que serão beneficiadas com a construção das cisternas.

Após ser constituída a comissão municipal dos projetos, são realizadas reuniões nas quais são relatadas as necessidades de cada comunidade e, conjuntamente, definidos os critérios para decidir quais serão as famílias beneficiadas. Segundo os entrevistados, este processo está isento de clientelismos. Nesse sentido, eles apresentaram alguns critérios que permeiam a escolha final dos beneficiários nas comunidades, a saber: agricultores cadastrados com baixa renda, pessoas beneficiadas pelo Programa Bolsa Família, famílias que tem como "chefe da família" a mulher, e famílias com crianças e idosos. De acordo com os relatos obtidos nas entrevistas, a ideia é que a água para consumo humano chegue primeiro a quem mais precisa.

Os entrevistados, de forma unânime, confirmaram que as cisternas de placas construídas nos projetos são consideradas uma inovação social, não apenas pelo benefício específico de obter água na cisterna, mas pelo processo de envolvimento e capacitação que o projeto desenvolve juntos aos agricultores, o que inclui as oficinas para obter uma consciência sobre gestão de água para produção de alimentos e gestão de recursos hídricos. Além disto, eles referenciaram que outra experiência de formação para os agricultores é o intercâmbio das experiências bem-sucedidas de manejo de água. Os agricultores que obtiveram êxitos nas suas ações em uma determinada comunidade apresentam-nas em outras localidades, para que haja um momento de troca de experiências e adaptações a suas realidades enquanto agricultores. Surgem, assim, espaços para novos conhecimentos, para valorização da cultura e do desenvolvimento comunitário local.

Nas ações de intercâmbio, os agricultores conhecem novas realidades, valorizam a troca de experiências e criam laços solidários entre eles. As experiências utilizadas para o intercâmbio são sistematizadas em formas de boletins que contam as histórias de vida das famílias e como seu conhecimento contribuiu para a melhoria da produção e para a qualidade de vida (DUQUE, 2008). Assim, os aspectos observados nos processos de intercâmbio de experiências nos projetos desenvolvidos pelo PATAC corroboram com Leal (2013), quando ressalta que estas iniciativas de capacitação são realizadas de forma coletiva, articuladas pelas organizações sociais envolvidas e pelos representantes das comunidades, representando a visão da coletividade no processo de busca pelo conhecimento.

Além disto, deve-se considerar que os projetos desenvolvidos pelo PATAC, a exemplo do projeto de construção de cisternas, vão além do benefício do produto envolvido, que neste caso é a água, mas se volta para um processo de emancipação dos atores locais, tendo, no centro, os próprios usuários dessas tecnologias (LEAL, 2013). Assim, fica entendido que as cisternas por si só não se caracterizam uma inovação social, mas a inserção do agricultor em todo o processo, desde o cadastramento até as capacitações e os intercâmbios realizados entre as comunidades, valorizando o saber popular. 


\subsection{NÍVEL ORGANIZACIONAL}

A inovação social analisada sob o prisma do nível organizacional busca captar a definição das estruturas organizacionais nas iniciativas, na forma de governança, e nos impactos no nível de aprendizagem coletiva (RODRIGUES, 2004). Nesse sentido, os resultados obtidos na etapa de coleta de dados possibilitaram inferir que o PATAC trabalha com um modelo de gestão integrado e participativo, com foco nas execuções de seus projetos sociais. Segundo relato dos entrevistados, dentro do processo de gestão do PATAC, a gestora executiva é responsável pela coordenação e sistematização dos projetos e pelas decisões políticas gerais, e o gestor técnico é responsável pelo acompanhamento da execução dos projetos. Porém, ressalta-se que a definição das ações operacionais faz parte de uma política democrática que conta com a participação dos integrantes da comissão municipal, composta por unidades gestoras, comissões municipais e representantes de grupos organizados da sociedade civil.

Um aspecto que foi destacado na execução dos projetos desenvolvidos pelo PATAC é a participação das famílias das comunidades beneficiadas nas discussões sobre seus direitos, do processo de formação enquanto cidadãos, da convivência com o semiárido e a responsabilidade que devem ter no uso da água. Nesse sentido, elas estão envolvidas em todas as etapas dos projetos, desde a mobilização, processo formativo e implantação do benefício. Desta forma, os processos de execução dos projetos acontecem de forma descentralizada e participativa, através da articulação de uma rede de atores envolvidos. No caso específico das cisternas, os agricultores beneficiados rompem com a dependência política que possuíam anteriormente e passam a ter acesso à água, iniciando um processo de construção de cidadania e autonomia na gestão de seus recursos hídricos.

Com base nesses resultados, verifica-se que os processos de mobilização social desenvolvidos no PATAC fazem parte de um programa de educação cidadã, na busca pela formação de uma rede social com o objetivo de alcançar soluções de maneira compartilhada para os problemas enfrentados. Os ganhos obtidos nesse processo estão além dos benefícios obtidos com a implantação de uma tecnologia social, mas também têm gerado reflexões sobre a vida comunitária, as formas de participação e organização popular, e novas formas de atrair outras políticas públicas, contribuindo para a transformação social do cotidiano das famílias (MEDEIROS; SILVEIRA; NEVES, 2010).

Assim, percebe-se que as estruturas que permeiam a nova configuração do semiárido possibilitaram novas formas de coordenação e divisão de trabalho, migrando de uma estrutura assistencialista para uma abordagem emancipatória. 


\subsection{NÍVEL INSTITUCIONAL}

É importante ressaltar que o envolvimento de um único ator não garante a inovação social. Este ator pode agir como uma condução necessária (embora não suficiente) para a contribuição de um processo de inovação, mas é necessário o desenvolvimento de parcerias intersetoriais para que a inovação social aconteça (DAVIES et al., 2012).

Estes aspectos podem ser observados nos exemplos dos programas P1MC (construção de cisternas de placa) e P1+2 (cisternas calçadão), que neste estudo são executados pelo PATAC no semiárido paraibano, mas que são programas do Governo Federal. A proposta da criação desses programas foi desenvolvida pela Articulação do Semi-Árido (ASA), contando com a participação de seus representantes em cada região, e entregues ao Governo Federal, em 1999, com o propósito de buscar mecanismo viável para colocá-la em prática. Vale esclarecer que a ASA é resultado da mobilização de diferentes atores que atuam na região, desde a década de 1970, na busca por alternativas para enfrentamento dos problemas da seca (TEODÓSIO, 2008).

De acordo com a gestora administrativa do PATAC, uma vez pautado esses projetos ao Governo Federal, realizou-se um levantamento das necessidades de primeira água das diversas regiões do semiárido brasileiro, a partir do estabelecimento de parcerias com as organizações sociais que estavam à frente da proposta inicial nas diversas localidades. Assim, deu-se início ao Programa Um Milhão de Cisternas (P1MC), em 1999 e intensificado a partir de 2003, com o propósito da universalização da "primeira água", ou a água para o consumo humano.

Esse exemplo de inovação no nível institucional reforça o argumento de que o envolvimento dos representantes da sociedade civil, tanto na esfera individual como coletiva, bem como das instituições públicas em projetos de cunho social, se relacionam ao engajamento em atividades para reforçar a legitimidade e a responsabilidade de instituições democráticas, como também contribuir com a coesão social na medida em que fornecem informações relevantes sobre as necessidades e condições locais, ajudando, assim, na tomada de decisão através da formulação de políticas públicas mais eficazes, e que possam beneficiar a localidade (DAVIES et al., 2012), bem como na própria execução dos projetos.

Assim, constatou-se que a ASA pauta a luta da água para o consumo humano para populações difusas. Nesse sentido, o gestor técnico entrevistado comentou que, uma vez aprovado e intensificado pelo Governo Federal em 2003, o Ministério do Desenvolvimento Social (MDS) passou a contratar entidades da sociedade civil para executar o programa. O entrevistado complementa que, com o sucesso da implementação das cisternas de Placas, a ASA lançou em 2007 o Programa Uma Terra e Duas Aguas (P1+2), que provê as cisternas de águas para a produção de alimentos. O projeto enviado ao Governo Federal pauta a água para a produção de alimentos, também com base em tecnologias sociais, a exemplo das cisternas calçadão e barreira trincheira, e melhorias nos tanques de pedra. 
Confirmando as informações obtidas na realização deste estudo, Dagnino (1994) afirma que o exercício democrático não ocorre apenas quando o Estado responde às reinvindicações da sociedade, mas quando os seus próprios sujeitos exercem a cidadania e se tornam agentes fundamentais para a conquista de seus direitos.

Ressalta-se, também, que a ampliação do projeto P1MC para o projeto P1+2 trouxe novos elementos para o processo de formação dos agricultores, e agora se inserem nas agendas de capacitação a produção de alimentos saudáveis sem o uso de agrotóxico, defensivos naturais, e sobre fertilidade do solo. Desta forma, entende-se que, para se tornar uma força de inovação e transformação na escala da sociedade, as iniciativas das organizações sociais não podem se isolar, pois as articulações entre Estado, empresas e sociedade civil levam a um estágio elevado de transformação social.

Assim, o crescente interesse em inovação social não deve ser entendido apenas como mecanismo isolado de satisfação das necessidades sociais, mas também como um mecanismo de transformação social que busca mudanças na configuração da sociedade como meio de promover a sustentabilidade e a qualidade de vida.

\section{CONSIDERAÇÕES FINAIS}

O objetivo deste trabalho foi de analisar as iniciativas de inovação social desenvolvidas pelo PATAC a partir do modelo proposto por Lévesque (2002). Considera-se que o PATAC é uma organização social que tem apoiado a implementação e execução de projetos sociais e criado alternativas para melhorar as condições dos agricultores familiares do semiárido paraibano, viabilizando a convivência daquela região com os problemas da seca e a inserção de tecnologias sociais como políticas públicas. Nesse sentido, foram observados os três níveis de análise apresentados no modelo: atores, organizacional e institucional.

Na perspectiva do nível de análise dos atores, percebeu-se que, no âmbito do PATAC, o programa de cisternas é um exemplo claro de inovação social pela mobilização de todos os atores envolvidos, pela valorização da cultura e da capacidade de aprendizado, promovendo o desenvolvimento social das comunidades onde os projetos são implementados.

Por outro lado, no nível organizacional, observou-se que, nos projetos geridos e executados pelo PATAC, são adotadas novas formas de gestão, com um caráter compartilhado, na busca do fortalecimento sociopolítico da organização dos agricultores a partir da gestão coletiva do conhecimento que eles compartilham entre si, o que simboliza o respeito mútuo, a valorização da cultura e da nova perspectiva de convivência com o semiárido. Neste nível de análise, evidenciou-se, ainda, um benefício que enfoca a criação de uma autoridade de poder para cumprir a função de atingir as metas e os objetivos dos programas sociais e identificar e mobilizar todos os recursos para sua realização. Neste sentido, a inovação social teve 
como alvo mudanças nas relações sociais, particularmente em relação à governança, para permitir que as necessidades sejam atingidas e também melhorar a participação dos grupos excluídos nos processos de tomada de decisão (ASSOGBA, 2010).

Por fim, no nível institucional, os resultados demonstram que o processo de inovação social envolvido nos projetos desenvolvidos pelo PATAC tem como função coordenar os diferentes setores da sociedade para manter redes de relacionamentos e cooperação para funcionamento dos projetos e programas sociais envolvidos, ou seja, é uma integração desta pluralidade de atores sociais, com o objetivo de aumentar o capital social, os níveis de aprendizado, e as capacidades de cada membro participante. Assim, do ponto de vista da integração social, a inovação social também deve fazer uma ligação entre diferentes níveis da estrutura da sociedade, para garantir o desenvolvimento social a nível local e regional (ASSOGBA, 2010).

Conclui-se, portanto, que as iniciativas de inovação social investigadas neste estudo contribuem para o desenvolvimento sustentável das localidades em que estão inseridas, na medida em que são identificadas e difundidas ações nas comunidades beneficiadas, gerando assim, significativos impactos socioeconômicos, a exemplo de criação de empregos, formação e qualificação dos indivíduos, crescimento pessoal (melhoria da autoestima e confiança), um sentido de comunidade, capital social, melhoria dos serviços de saúde e educação e maior engajamento cívico (SEYFANF; SMITH, 2007).

Desta forma, os níveis de análise apresentados neste artigo serviram como uma ferramenta de tradução do conceito teórico e abstrato da inovação social, cuja proposta ajuda a estabelecer uma perspectiva abrangente sobre a criação do valor social e contribuir para que os atores (sociais, institucionais e organizacionais) legitimem suas atividades de inovação social, embasados em dados que caracterizam a realidade social e promovam o desenvolvimento sustentável. Porém, vale ressaltar que o tema em análise carece da elaboração de elementos teóricos e objetivos em termos mais pragmáticos, que possibilitem operacionalizar melhor o conceito e avaliar os impactos que a implantação dessas iniciativas de inovação social geram nas localidades beneficiadas em termos de melhoria na qualidade vida e contribuição para a sustentabilidade.

\section{REFERÊNCIAS}

ANDRÉ, I.; ABREU A. Dimensões e espaços da inovação social. Finisterra, a. 41, v. 81, p. 121-141, 2006.

ASSOGBA, Y. Théorie systémique de l'action sociale et innovation sociale. Alliance de recherche université-communauté/innovation sociale et développement des communautés (AURC-ISDC), Université du Québec en Outaouais (UQO). Recherches, n. 31, mar. 2010. 
BARDIN, L. Análise de conteúdo. Porto: Edições 70, 2008.

BIGNETTI, L. P. As inovações sociais: uma incursão por ideias, tendências e focos de pesquisa. Ciências Sociais Unisinos, v. 47, n. 1, p. 3-14, jan./abr. 2011.

BITTENCOURT, B; RONCONI, L. Políticas de Inovação social e Desenvolvimento: O caso da Bolsa de Terras. Revista Administração Pública, v. 50, n. 5, p. 795-818, 2016.

BOCK, B. Rural marginalization and the role of social innovation: a turn towards nexogenous development and rural reconnection. Sociologia Ruralis, 2016.

BODSTEIN, R. et al. Avaliação da implementação do programa de desenvolvimento integrado em Manguinhos: impasses na formulação de uma agenda local. Ciência \& Saúde Coletiva, v. 9, n. 3, p. 593-604, 2004.

CAJAIBA-SANTANA, G. Social innovation: Moving the field forward. A conceptual framework. Technological Forecasting and Social Change, v. 82, 42-51, 2014.

CAULIER-GRICE, J. et al. Defining Social Innovation. A deliverable 1.1 of the project: 'The theoretical, empirical and policy foundations for building social innovation in Europe' (Tepsie), European Commission. Brussels: European Commission, 2012.

CARON, A. Inovação Social e o Papel da Indústria. In: FARFUS, D.; ROCHA, M. C. de S. Inovações Sociais. Curitiba: SESI/SENAI/IEL/UNINDUS, 2007. (Coleção Inova; V. II).

CLOUTIER, J. Qu' est-ce que l'innovation sociale? Cahier de recherche du CRISES, n. ET0314, Montreal: UQAM, 2003.

CHAMBON, J.; DAVID, A.; DEVEVEY, J. Les Innovations sociales. Que sais-je Paris: Presses universitaires de France, 1982.

CORREIA, S. É. N.; OLIVEIRA, V.; GOMEZ, C. R. P. Dimensions of social innovation and the roles of organizational actor: the proposition of a framework. RAM. Revista de Administração Mackenzie, v. 17, n. 6, 102-133, 2016.

COMISSÃO EUROPÉIA. Iniciativa de empreendedorismo social: construir um ecossistema para promover as empresas sociais no centro da economia e da inovação social. Bruxelas, 2011.

DAGNINO, E. Os movimentos sociais e a emergência de uma nova noção de cidadania. In: DAGNINO, Evelina (Org.). Anos 90: política e sociedade no Brasil. São Paulo: Editora brasiliense, 1994.

DAGNINO, R. P. A tecnologia social no Brasil. In. DAGNINO, Renato Peixoto (Org.). Tecnologia social: ferramenta para construir outra sociedade. Campinas, SP: IG/UNICAMP, 2009. 
DAVIES, A. et al. Mapping citizen engagement in the process of social innovation. The Young Fundation, September, 2012.

DAVIES, Anna; et al. Mapping citizen engagement in the process of social innovation. The Young Fundation, September, 2012.

DUQUE, G. "Conviver com a seca": contribuição da Articulação do Semi-árido/ASA para o desenvolvimento sustentável. Desenvolvimento e Meio Ambiente, n. 17, p. 133-140, jan./jun. 2008.

EVA, B. et al. Blueprint of Social Innovation Metrics: Contributions to an understanding of the opportunities and challenges of social innovation measurement. TEPSIE, University of Heidelberg, 2013.

GENRO, T. Porto da cidadania: a esquerda no governo de Porto Alegre. Porto Alegre: Artes e Ofícios, 1997, p. 267.

GODOY, A. S. Introdução à pesquisa social qualitativa e suas possibilidades. Revista de Administração de Empresas, v. 35, n. 2, mar./abr. 1995, p. 57-63.

HOWALDT, J.; DOMANSKI, D.; KALETKA, C. Social Innovation: towards a new innovation paradigm. RAM. Revista de Administração Mackenzie, v. 17, n. 6, 2016, p. 20-44.

HOWALDT, J.; KOPP, R.; SCHWARZ, M. Social Innovations as Drivers of Social Change-Exploring Tarde's Contribution to Social Innovation Theory Building. New Frontiers in Social Innovation Research, p. 29-51. UK: Palgrave Macmillan UK, 2015.

HULGARD, L.; FERRARINI, A. V. Inovação social: rumo a uma mudança experimental na política pública?

Ciências Sociais Unisinos, v. 46, n. 3, p. 256-263, 2010.

ITS - INSTITUTO DE TECNOLOGIA SOCIAL. Caderno tecnologia social: conhecimento e cidadania. ITS, fev. 2007.

KLEIN, J.; HARRISSON, D. L'innovation sociale: Émergence et effets sur la transformation des societies. Canada: Press de I'Université du Québec, 2007.

LALLEMAND, D. Les défis de l'innovation sociale. Collection Actions sociales Société; Issy-les-Moulineaux: ESF, 2001.

LASSANCE JR., E.; PEDREIRA, S. Tecnologias sociais e políticas públicas. Tecnologia social: uma estratégia para o desenvolvimento. Rio de Janeiro: Fundação Banco do Brasil, 2004.

LEAL, A. K. Cisterna de placa: uma tecnologia social para a convivência com o semiárido. V Simpósio de Tecnologia em Meio Ambiente e Recursos Hídricos - FATEC. Anais... São Paulo, 2013. 
LÉVESQUE, B. Les entreprises d'économie sociale, plus porteuses d'innovations sociales que les autres? In: COLLOQUE DU CQRS AU CONGRES DE L’ACFAS, 2001, Montreal, Cahiers du CRISES, v. 0205, 2002.

MEDEIROS, J.; SILVEIRA, S.; NEVES, R. Agua e cidadania no semiárido brasileiro: A experiência do Programa Um Milhão de Cisternas rurais (P1MC) da ASA Brasil. VII Congresso Latinoamericano de Sociologia Rural. Anais... Porto de galinhas, 2010.

MOULAERT, F. et al. Towards Alternative Model(s) of Local Innovation. Urban Studies, n. 42, v. 11, 2005, p. 1.969-90.

MOULAERT, F. Social Innovation: Institutionally Embedded, Territorially (Re)Produced. MacCallum, D.; Moulaert, F.; Hillier, J.; Vicari Haddock, S. Social Innovation and Territorial Development, Farnham: Ashgate, 2009.

MOULAERT, F.; et al. Social Innovation: Collective action, social learning and transdisciplinary research. Katarsis, 2010.

MULGAN, G.; et al. Social innovation. What it is, why it matters and how it can be accelerated. London: Young Foundation, 2007.

NILSSON, W. O. Social Innovation: An Exploration of the literature. McGill University, 2003.

OECD. Social Entrepreneurship in Social Innovation. SME's Entrepreneurship and innovation, p. 185-215. Paris, 2010.

OLIVEIRA, N.; SILVA, T. Inovação social e tecnologias sociais sustentáveis em relacionamentos intercooperativos: um estudo exploratório no CREDITAG - RO. Revista Adm, UFSM, Santa Maria, v. 5, n. 2, p. 277-295, mai./ago. 2012.

PATAC - Programa de articulação de tecnologias apropriadas para comunidades. Disponível em: <http:// www.patacparaiba.blogspost.com.br/p/patac.html . Acesso em: 01 ago. 2014.

PHILLS JR., J. A.; DEIGMEIER, K.; MILLER, D. T. Rediscovering social innovation. Stanford Social Innovation Review, Fall, p. 34-43, 2008.

POL, E.; VILLE, S. Social innovation: Buzz word or enduring term? Journal of Socio-Economics, dec. 2009.

RODRIGUES. A. L. Modelos de Gestão e inovação social em organizações sem fins lucrativos: um estudo comparativo de casos no Brasil e no Quebec. Tese (Doutorado) - Fundação Getulio Vargas, São Paulo, 2004.

RODRIGUES, A. L. Organizações sociais: Um estudo de caso sobre possibilidades e limitações da geração de inovação social pela OSESP. Anais... VI ENAPEGS, São Paulo, 21 a 23 de maio de 2012. 
RODRIGUES, I.; BARBIERI, J. C. A emergência da tecnologia social: revisitando o movimento da tecnologia apropriada como estratégia de desenvolvimento sustentável. Revista de Administração Pública, Rio de Janeiro, v. 42, n. 6, p. 1069-94, nov./dez. 2008.

RICHARDSON, R. J. Pesquisa Social: métodos e técnicas. São Paulo: Saraiva, 1985.

RUIZ, J. E., Inovação Social: desafios e estratégias para o desenvolvimento inclusivo do território. V Diálogo Regional. Anais... Recife, mai. 2012.

SEYFANG, G.; SMITH, A. Grassroots Innovations for Sustainable Development: Towards a New Research and Policy Agenda. Environmental Politics, v. 16, n. 4, p. 584-603, aug. 2007.

SHARRA, R.; NYSSENS, M. Social Innovation: An Interdisciplinary and Critical Review of the Concept. Louvain, Belgium: Université Catholique de Louvain, 2010.

TARDIF, C.; HARRISSON, D. Complémentarité, convergence et transversalité: la conceptualisation de l'innovation sociale au CRISES. In: CRISES. Centre de Recherche Sur Les Innovations Sociales. Cahiers du CRISES. Québec, 2005.

TAYLOR, JAMES B. Introducing Social innovation. The Journal of Applied Behavioral Science, v. 6, n. 1, p. 69-77, mar. 1970.

TEODÓSIO, A. S. Parcerias Trissetoriais na esfera pública: implicações, impasses e perspectivas acerca da provisão de políticas sociais em três experiências. Tese (Doutorado) - Escola de Administração de Empresas de São Paulo, São Paulo/SP, 2008.

YIN, R. Estudo de caso: Planejamento e métodos. Porto Alegre: Bookman, 2005. 